Insbesondere mit Blick auf die Europawahl 2009 scheint es geboten, eine (europäische) Institution zu beauftragen, die die amtlichen Ergebnisse der EP-Wahlen sammelt, auswertet und zur Verfügung stellt. Die nationalen Ergebnisse und Beteiligungen der Wahlen zum Europäischen Parlament sind dabei der Ausgangspunkt. Wünschenswert sind insbesondere auch regionale / lokale Wahlergebnisse, um Abweichungen vom nationalen Trend feststellen zu können. Zudem sind die Resultate der nationalen Hauptwahlen zu berücksichtigen, da die Europawahlergebnisse am besten als Abweichung von diesen interpretiert werden können. Angaben zum Wahlsystem und Wahlverfahren (Wahlpflicht, Wahltermin) sowie die Information, ob die EP-Wahl mit der nationalen Hauptwahl oder mit einer anderen, wichtigen Nebenwahl zusammenfällt, würden zudem eine erste Einordnung der Ergebnisse und Beteiligungsraten im internationalen Vergleich ermöglichen.

\title{
Ökonomisches Wählen: Zum Einfluss von Wahrnehmungen der allgemeinen Wirtschaftslage auf das Abschneiden der Bundesregierungsparteien bei Landtagswahlen*
}

\author{
Ivar Krumpal und Adrian Vatter
}

Politikwissenschaftler waren seit jeher an der engen Verflechtung zwischen Wirtschaft und Politik interessiert. Entsprechend häufig werden diese komplexen Zusammenhänge untersucht. Insbesondere im Bereich der empirischen Wahlforschung haben sich auf dem Gebiet des ökonomischen Wählens („Economic Voting“) recht robuste Trends auf der Aggregatebene abgezeichnet. Dabei verdichten sich die Befunde zu einem relativ stabilen Zusammenhang zwischen der allgemeinen Konjunkturentwicklung eines Landes und der Popularitäts- beziehungsweise Stimmentwicklung der jeweils amtierenden Regierungsparteien. ${ }^{1}$ Demnach ist mit überproportional hohen Stimmenverlusten besonders dann zu rechnen, wenn zum Zeitpunkt einer Wahl die wirtschaftliche Entwicklung negativ ist.

Allerdings zeichnen sich diese auf Aggregatdaten beruhenden Untersuchungen für Deutschland durch theoretische und methodische Schwächen aus. So fehlt ihnen in der Regel eine mikrotheoretische Fundierung ihrer Hypothesen, und die empirischen Ergebnisse unterliegen der bekannten Gefahr eines ökologischen Fehlschlusses. Die bisherigen Aggregatdatenstudien zum ökonomischen Wählen enthalten meist nur implizite Annahmen darüber, wie der einzelne Wähler seine subjektive wirtschaftliche Situation konkret wahrnimmt und welches Entscheidungsverhalten daraus resultiert. Zudem wird vernachläs-

* Für zahlreiche konstruktive Diskussionen und kritische Anmerkungen danken wir Heiko Rauhut, Thomas Voss und Wolfgang Langer. Der Beitrag ist teilweise im Rahmen des von der DFG geförderten Forschungsprojekts „Demokratiemuster in den deutschen Bundesländern“ entstanden, das von Markus Freitag und Adrian Vatter bearbeitet wird.

1 Michael S. Lewis-Beck / Mary Stegmaier, Economic Determinants of Electoral Outcomes, in: Annual Review of Political Science, 3. Jg. (2000), S. 183 - 219; Brian J. Gaines / Christophe Crombez, Another Look at Connections Across German Elections, in: Journal of Theoretical Politics, 16. Jg. (2004), S. $289-319$. 
sigt, dass der direkte Schluss von statistischen Zusammenhängen der Aggregatebene auf individuelles Verhalten problematisch ist.

Aus diesem Grund soll hier erstmalig für das Wählerverhalten in der Bundesrepublik Deutschland die These vom ökonomischen Wählen anhand von Individualdaten im $\mathrm{Zu}-$ sammenhang mit dem Abschneiden der Bundesregierungsparteien bei Landtagswahlen ${ }^{2}$ überprüft werden. Während frühere Arbeiten zumeist die Popularitätskurven beziehungsweise die Stimmenanteile der Regierungsparteien als eine Funktion von makroökonomischen Indikatoren (wie reales BIP etc.) modellieren, legen aktuellere internationale Studien vermehrt den Schwerpunkt auf die Zusammenhänge zwischen individueller Wahlabsicht und allgemeiner, subjektiv wahrgenommener Wirtschaftslage. ${ }^{3}$ Im letzteren Fall wird überwiegend auf der Individualebene argumentiert und mit Mikrodaten gearbeitet. An diese Forschungsperspektive knüpft der vorliegende Beitrag an und präsentiert für das Wahlverhalten im bundesdeutschen Mehrebenensystem einen direkten empirischen Test der Economic Voting-Hypothese mit Mikrodaten. Die im Zentrum stehende Forschungsfrage lautet dabei: Wie stark wirkt die subjektive Wahrnehmung der allgemeinen wirtschaftlichen Lage auf die individuellen Wahlpräferenzen bei Landtagswahlen? Gemäß der Theorie des ökonomischen Wählens ist es bei unzufriedenen Regierungsanhängern wahrscheinlicher, dass sie bei Landtagswahlen ihren Parteien den Rücken kehren und stattdessen eine Partei aus dem Lager der Bundesopposition wählen.

Betrachtet man zunächst einfache Kennziffern der amtlichen Wahlstatistik, so deuten diese im untersuchten Zeitraum (1995 bis 2002) auf klare Stimmenverluste der Bundesregierungsparteien bei Landtagswahlen hin. ${ }^{4}$ Bei den 27 Landtagswahlen mussten die jeweils amtierenden Bundesregierungsparteien relativ zu ihren Wahlergebnissen bei den Bundestagswahlen im Durchschnitt 12,9 Prozent ihrer erwarteten Stimmen einbüßen. ${ }^{5}$ Diese Schwäche führte dazu, dass die Bundesregierung einer Oppositionsmehrheit im Bundesrat gegenüberstand, die in der Lage war, zustimmungsbedürftige Gesetze zu verhindern. ${ }^{6}$ Dies

2 Das Abschneiden der Bundesregierungsparteien bei Landtagswahlen ist ebenso ein zentrales Thema in Heft 3/2007 der ZParl, vgl. Oscar W. Gabriel / Everhard Holtmann, Ober sticht Unter? Zum Einfluss der Bundespolitik auf Landtagswahlen: Kontext, theoretischer Rahmen und Analysemodelle, in: ZParl, 38. Jg. (2007), H. 3, S. 445 - 462. Die hier vorliegende Studie ergänzt diese Beiträge, legt allerdings den Analysefokus auf die bisher nur relativ wenig erforschte ökonomische Dimension.

3 Ramon M. Alvarez / Jonathan Nagler, Economics, Issues and the Perot Candidacy: Voter Choice in the 1992 Presidential Elections, in: American Journal of Political Science, 39. Jg. (1995), S. 714 - 744; André Blais / Mathieu Turgeon / Elisabeth Gidengi / Neil Nevitte / Richard Nadeau, Which Matters Most? Comparing the Impact of Issues and the Economy in American, British and Canadian Elections, in: British Journal of Political Science, 34. Jg. (2004), S. 555 - 563.

4 Die Berechnungen der relativen Stimmenverluste der Bundesregierungsparteien bei Landtagswahlen folgen dem von Reiner Dinkel vorgeschlagenen Verfahren. Hierbei wurden bei den jeweils betrachteten Landtagswahlen in einem gegebenen Bundesland die Wahlergebnisse der zum Landtagswahlzeitpunkt amtierenden Bundesregierungsparteien in Relation gesetzt zu ihren Stimmenanteilen bei den beiden zeitlich benachbarten Bundestagswahlen. Vgl. Reiner Dinkel, Der Zusammenhang zwischen Bundes- und Landtagswahlergebnissen, in: PVS, 18. Jg. (1977), S. 348 - 359.

5 Hierbei wiederum fielen bei den Landtagswahlen in Ostdeutschland die relativen Stimmenverluste mit einem Mittelwert von 24,3 Prozent $(\mathrm{n}=10)$ stärker aus als in den alten Bundesländern. Hier lag der mittlere Stimmenverlust bei 6,2 Prozent $(n=17)$.

6 So verlor im Jahre 1999 die damals amtierende rot-grüne Bundesregierung unter Gerhard Schröder bereits vier Monate nach ihrem deutlichen Wahlsieg bei den Bundestagswahlen am 27. Sep- 
hat im institutionell eng verflochtenen System der Bundesrepublik beträchtliche Konsequenzen wie die Vorherrschaft der Oppositionsparteien im Bundesrat, die damit verknüpfte Gefahr einer Blockade gesetzgeberischer Entscheidungsprozesse und den Verlust der Zurechenbarkeit von politischer Verantwortung.

Ausdrücklich hervorzuheben ist, dass es sich beim hier untersuchten ökonomischen Wählen und beim von Reiner Dinkel ${ }^{7}$ herausgearbeiteten zyklischen Midterm-Verlust um zwei klar voneinander zu trennende Phänomene handelt. Der Midterm-Verlust kann mit wirtschaftlichen Entwicklungen zusammenhängen, muss es aber nicht. Wenn eine pessimistische Einschätzung der wirtschaftlichen Lage den Stimmenverlust der Regierungsparteien bei Landtagswahlen verursacht, führt dies noch nicht automatisch zu einem zyklischen Midterm-Verlust. Zudem gibt es weitere Ursachen, die zu schlechteren Wahlergebnissen der Bundesregierungsparteien bei Landtagswahlen führen können, so etwa ein durch externe Umstände erzwungener Wechsel der politischen Positionen.

\section{Theoretische Grundlagen, Forschungsstand und Hypothesen}

\subsection{Die Grundlagen des Economic Voting-Ansatzes}

$\mathrm{Zu}$ den ersten Anwendungen ökonomischer Theorien auf die Analyse politischer Prozesse zählen die Arbeiten von Joseph A. Schumpeter und Anthony Downs. ${ }^{8}$ Im Sinne einer ökonomischen Tauschlogik modellierte Downs die moderne westliche Demokratie als einen politischen Markt, auf dem die Parteien als Anbieter und die Wähler als Nachfrager auftreten. Hierbei bieten die Parteien ihre politischen Programme an und werben so um die Stimmen der Wähler. Dabei postuliert das Axiom der rationalen Wahl, dass ein Bürger stets diejenige Partei wählen wird, deren Programm ihn in der Zukunft den höchsten Nettonutzen erwarten lässt. ${ }^{9}$

tember 1998 ihre Mehrheit im Bundesrat. Entscheidend hierfür war die Landtagswahl am 7. Februar in Hessen, welche überraschenderweise an die CDU verloren ging. Davor hatte auch schon die Regierung unter Helmut Kohl Anfang der 1990er Jahre ihre Vormachtstellung in der Länderkammer eingebüßt.

7 Reiner Dinkel, a.a.O. (1977); ders., Zur Gesetzmäßigkeit der Trendverschiebungen zwischen Landtags- und Bundestagswahlen, in: ZParl, 12. Jg. (1981), H. 1, S. 135 - 139; ders., Landtagswahlen unter dem Einfluss der Bundespolitik: Die Erfahrung der letzten Legislaturperioden, in: Jürgen W. Falter / Hans Rattinger / Klaus G. Troitzsch (Hrsg.), Wahlen und politische Einstellungen in der Bundesrepublik Deutschland, Frankfurt am Main 1989, S. 253 - 262. So konnte Dinkel für die Zeit vor der Wende einen zyklischen Zusammenhang zwischen den relativen Stimmenverlusten der amtierenden Regierungsparteien und der zeitlichen Position einer Landtagswahl mit Aggregatdaten nachweisen. Gemäß seinem Modell sind die relativen Stimmenverluste der amtierenden Bundesregierungsparteien bei Landtagswahlen in der Mitte einer Legislaturperiode am höchsten. Dagegen wird für die Zeit kurz vor der Bundestagswahl wegen der Mobilisierung durch den Wahlkampf beziehungsweise kurz nach der Bundestagswahl aufgrund einer so genannten Honeymoon-Periode ein lediglich geringer Stimmenverlust vorhergesagt. Zu einer weiterführenden Diskussion vgl. Simone Burkhart, Parteipolitikverflechtung. Über den Einfluss der Bundespolitik auf Landtagswahlentscheidungen von 1976 bis 2002, in: PVS, 46. Jg. (2005), S. $14-38$.

8 Joseph A. Schumpeter, Capitalism, Socialism and Democracy, New York 1942; Anthony Downs, An Economic Theory of Democracy, New York 1957.

9 Vgl. Anthony Downs, a.a.O., S. 36 - 39. 
Gemäß dieser theoretischen Tradition wurden zunächst seit Beginn der 1970er Jahre in der angelsächsischen Politikwissenschaft umfangreiche empirische Studien zum ökonomischen Wählen anhand von Aggregatdaten durchgeführt. ${ }^{10}$ Das zentrale Problem dieser Vorgehensweise besteht darin, dass die zugrunde liegende individuelle Entscheidungssituation in den meisten Fällen nicht statistisch modelliert wurde. Vielmehr wurden aggregierte Daten verwendet, die den Zusammenhang mit individuellem Wahlverhalten nur hypothetisch annehmen konnten: „Clearly, the inferences that can be made about individual voting decisions from aggregate data are quite limited."11 Kurz: Aus einer Zeitreihe von aggregierten Wahlstatistiken und Verläufen makroökonomischer Kennwerte auf die zugrunde liegenden individuellen Entscheidungsmotive und das Verhalten des einzelnen Wählers zu schließen, ist problematisch. ${ }^{12}$

Spätere Arbeiten erkannten diese Problematik und verlagerten ihren Schwerpunkt auf die Mikroebene. ${ }^{13}$ Im Gegensatz zu den oben skizzierten Ansätzen stehen hier nicht mehr die statistischen Zusammenhänge zwischen Verläufen aggregierter volkswirtschaftlicher Kenngrößen und den Entwicklungen amtlicher Wahlstatistiken im Zentrum des Interesses. Stattdessen modellieren Mikrostudien die individuell formulierte Wahlabsicht als eine

10 Vgl. Charles A. E. Goodhart / Rajendra J. Bhansali, Political Economy, in: Political Studies, 18. Jg. (1970), S. 43 - 106; Gerald H. Kramer, Short-term Fluctuations in U.S. Voting Behavior, 1896 - 1964, in: American Political Science Review, 65. Jg. (1971), S. 131 - 143; William L. Miller I Myles Mackie, The Electoral Cycle and the Asymmetry of Government and Opposition Popularity: An Alternative Model of the Relationship between Economic Conditions and Political Popularity, in: Political Studies, 21. Jg. (1973), S. 263 - 279; Edward R. Tufte, Determinants of the Outcomes of Midterm Congressional Elections, in: American Political Science Review, 69. Jg. (1975), S. $812-826$.

11 Federico Ferrara / Timo J. Weishaupt, Get Your Act Together: Party Performance in European Parliament Elections, in: European Union Politics, 5. Jg. (2004), S. 283 - 306. Von einem „ökologischen Fehlschluss" spricht man, wenn fälschlicherweise von Korrelationen auf der Aggregatebene auf Zusammenhänge auf der individuellen Ebene geschlossen wird; vgl. hierzu William S. Robinson, Ecological Correlations and the Behavior of Individuals, in: American Sociological Review, 15. Jg. (1950), S. 351 - 357, und Gary King / Robert O. Keohane / Sidney Verba, Designing Social Inquiry: Scientific Inference in Qualitative Research, Princeton / New Jersey 1994, S. 30. Michael S. Lewis-Beck / Mary Stegmaier, a.a.O., diskutieren die Problematik direkt im Zusammenhang mit dem Economic Voting-Ansatz.

12 Allerdings wurde in der Vergangenheit bei einigen speziellen Fragestellungen auch argumentiert, dass sich ökonomische Effekte auf Wahlentscheidungen mit Aggregatdaten besser erfassen ließen als mit Individualdaten. Vgl. hierzu Gerald H. Kramer, The Ecological Fallacy Revisited: Aggregate- versus Individual-level Findings on Economics and Elections, and Sociotropic Voting, in: American Political Science Review, 77. Jg. (1983), S. 92 - 111. Die neuere Debatte, beispielhaft durch die Kontroverse zwischen Gary King und David A. Freedman vertreten, mahnt jedoch weiterhin zur Vorsicht: „Freedman et al. (1998) are right about one point: if one can avoid making inferences about individuals from aggregate data, then one should do so. And of course, valid survey data make ecological inferences superfluous." Gary King, The Future of Ecological Inference Research: A Comment on Freedman et al., in: Journal of the American Statistical Association, 94. Jg. (1999), S. 352 - 357 (mit Diskussion); David A. Freedman / Stephen P. Klein / Michael Ostland / Michael R. Roberts, A Solution to the Ecological Inference Problem, in: Journal of the American Statistical Association, 93 Jg. (1988), S. 1518 - 1522 (Review).

13 Morris P. Fiorina, Economic Retrospective Voting in American National Elections: A Micro-analysis, in: American Journal of Political Science, 22. Jg. (1978), S. 426 - 443; Roderick D. Kiewiet, Macroeconomics and Micropolitics: The Electoral Effects of Economic Issues, Chicago 1983. 
Funktion der subjektiv wahrgenommenen Wirtschaftslage. Hierbei werden zwei Varianten unterschieden:

(1) Retrospektive Variante: Auf den Fall der Landtagswahlen in Deutschland angewendet, geht die Annahme des retrospektiven ökonomischen Wählens davon aus, dass die Chancen auf eine Wiederwahl der die Bundesregierung tragenden Parteien steigen, wenn die Bürger eine Verbesserung der wirtschaftlichen Lage seit der letzten Bundestagswahl wahrnehmen. Hier wird also ein „Referendum“ der Wähler über die bisherigen wirtschaftspolitischen Leistungen der Regierungsparteien in der laufenden Wahlperiode angenommen. Zu den ersten Ansätzen einer Theorie der retrospektiven ökonomischen Wahl gehört die Arbeit von Vladimir O. Key ${ }^{14}$; eine systematische Ausformulierung und erste mikroanalytische Anwendungen findet man bei Morris P. Fiorina ${ }^{15}$. Beide Autoren gründeten ihre Konzepte auf das US-amerikanische Wählerverhalten. Neuere Studien legten Gregory B. Markus sowie Ramon M. Alvarez und Jonathan Nagler vor. ${ }^{16}$

(2) Prospektive Variante: Die Hypothese des prospektiven ökonomischen Wählens geht dagegen von einer in die Zukunft gerichteten Wählerperspektive aus. Gemäß dieser Variante stimmen Bürger bei Landtagswahlen gegen die Bundesregierung, wenn sie die zukünftige wirtschaftliche Entwicklung pessimistisch einschätzen. Dieser Auffassung liegt das Konzept des Wahlverhaltens rationaler Individuen zugrunde, wie es bereits Anthony Downs formulierte. Empirische Tests für die USA lassen sich bei Brad Lockerbie und David. J. Lanoue finden. ${ }^{17}$ Auch die folgende Analyse konzentriert sich auf diese Variante, da hierzu bisher wenige empirische Studien vorliegen.

Weiterhin wird in der Literatur zwischen Wahrnehmungen der persönlichen wirtschaftlichen Situation („pocketbook voting“) und Bewertungen der allgemeinen ökonomischen Lage („sociotropic voting“) unterschieden. Hier konnten Michael S. Lewis-Beck und Mary Stegmaier ${ }^{18}$ in ihrer bis heute umfangreichsten Literaturübersicht zu Economic Voting zeigen, dass die individuelle Wahlentscheidung viel stärker durch die Wahrnehmungen der allgemeinen wirtschaftlichen Situation beeinflusst wird als durch die Beurteilung der eigenen ökonomischen Verhältnisse.

\subsection{Die empirische Relevanz der Economic Voting-Hypothese in der internationalen Wahlforschung}

Zu den US-amerikanischen Pionierarbeiten über ökonomische Determinanten und deren Einfluss auf die Stimmenanteile der Partei des Präsidenten bei den US-Kongresswahlen gehört der Artikel von Gerald H. Kramer. ${ }^{19}$ Er stellt einen signifikanten Effekt des realen

14 Vladimir O. Key, The Responsible Electorate, New York 1966.

15 Morris P. Fiorina, Retrospective Voting in American National Elections, New Haven 1981; ders., a.a.O. (1978).

16 Gregory B. Markus, The Impact of Personal and National Economic Conditions on Presidential Voting, 1956 - 1988, in: American Journal of Political Science, 36. Jg. (1992), S. 829 - 834; Ramon M. Alvarez / Jonathan Nagler, a.a.O., S. 714.

17 Brad Lockerbie, Prospective Voting in Presidential Elections, 1956 - 1988, in: American Politics Quarterly, 20. Jg. (1992), S. 308 - 325; David J. Lanoue, Retrospective and Prospective Voting in Presidential-Year Elections, in: Political Research Quarterly, 47. Jg. (1994), S. 193 - 205.

18 Michael S. Lewis-Beck / Mary Stegmaier, a.a.O.

19 Gerald H. Kramer, a.a.O. 
durchschnittlichen Einkommens pro Kopf auf das Abschneiden der jeweils amtierenden US-Regierung bei den Kongresswahlen fest: „In quantitative terms, a $10 \%$ decrease in per capita real personal income would cost the incumbent administration 4 or 5 percent of the congressional vote, other things being equal." 20 Anfang der 1990er Jahre stellte Robert S. Erikson ${ }^{21}$ nach einer kritischen Auseinandersetzung mit früheren Studien von Edward $R$. Tufte 22 einen ökonomischen Effekt grundsätzlich in Frage; die Mehrzahl der aktuelleren empirischen Untersuchungen ${ }^{23}$ stützt in der Regel jedoch die These des Economic Voting: „For all democratic nations that have received a reasonable amount of study, plausible economic indicators, objective or subjective, can be shown to account for much of the variance in government support." ${ }^{4} 4$ Während die Anwendung von Aggregatdaten in der internationalen Wahlforschung eine lange Tradition hat, stellen empirische Untersuchungen zur Economic Voting-Hypothese mit Individualdaten ein vergleichsweise junges Phänomen dar. ${ }^{25}$

\subsection{Zum Einfluss des ökonomischen Wählens auf die Stimmenverluste der Bundesregie- rungsparteien bei Landtagswahlen}

Neuere Makrostudien zu Einflüssen volkswirtschaftlicher Faktoren auf das Abschneiden der Bundesregierungsparteien bei Landtagswahlen haben Susanne Lohmann und andere, Brian J. Gaines und Christophe Crombez vorgelegt. ${ }^{26}$ Während Lohmann und andere nach ihrer

20 Ebenda, S. 141. Vgl. hierzu auch die Kritik an der Studie von Arcelus und Meltzer und die anschließende Replik durch Goodman und Kramer; Francisco Arcelus / Allan H. Meltzer, The Effect of Aggregate Economic Variables on Congressional Elections, in: American Political Science Review, 69. Jg. (1975), S. 1232 - 1269; Saul Goodman / Gerald H. Kramer, The Effect of Aggregate Economic Conditions on Congressional Elections: Comment, in: American Political Science Review, 69. Jg. (1975), S. $1255-1265$.

21 Robert S. Erikson, Economic Conditions and the Congressional Vote: A Review of the Macrolevel Evidence, in: American Journal of Political Science, 34. Jg. (1990), S. 373 - 407.

22 Edward R. Tufte, a.a.O. (1975); ders., Political Control of the Economy, Princeton 1978.

23 Steven D. Levitt, An Empirical Test of Competing Explanations for the Midterm Gap in the U.S. House, in: Economics \& Politics, 6. Jg. (1994), S. 25 - 37; Roderick D. Kiewiet / Michael Udell, Twenty-five years after Kramer: An assessment of economic retrospective voting based upon improved estimates of income and unemployment, in: Economics \& Politics, 10. Jg. (1998), S. 219 - 248; Karen L. Remmer / Francois Gélineau, Subnational Electoral Choice - Economic and Referendum Voting in Argentina, 1983-1999, in: Comparative Political Studies, 36. Jg. (2003), S. 801 - 821; Francisco J. Veiga / Linda G. Veiga, Popularity Functions, Partisan Effects and Support in Parliament, in: Economics \& Politics, 16. Jg. (2004), S. $101-115$.

24 Michael S. Lewis-Beck / Mary Stegmaier, a.a.O., S. 211.

25 Lonna R. Atkeson / Randall W. Partin, Economic and Referendum Voting: A Comparison of Gubernatorial and Senatorial Elections, in: American Political Science Review, 89. Jg. (1995), S. 99 - 107; Thomas J. Rudolph / Tobin J. Grant, An Attributional Model of Economic Voting: Evidence from the 2000 Presidential Election, in: Political Research Quarterly, 55. Jg, (2002), S. 805 - 823; Harold D. Clarke / Marianne C. Stewart, Economists and electorates: The subjective economy of governing party support in Canada, in: European Journal of Political Research, 29. Jg. (1996), S. 191 - 214; Michael S. Lewis-Beck, Who's the chef? Economic voting under a dual executive, in: European Journal of Political Research, 31 Jg. (1997), S. 315 - 325; André Blais I Mathieu Turgeon / Elisabeth Gidengi / Neil Nevitte / Richard Nadeau, a.a.O.

26 Susanne Lohmann / David W. Brady / Douglas Rivers, Party Identification, Retrospective Voting, and Moderating Elections in a Federal System: West Germany, 1961 - 1989, in: Comparative Political Studies, 30. Jg. (1997), S. 420 - 449; Brian J. Gaines / Christophe Crombez, a.a.O. 
Analyse für die Jahre von 1961 bis 1989 nur einen vergleichsweise unbedeutenden Effekt der volkswirtschaftlichen Entwicklung auf die Stimmenverluste der Bundesregierungsparteien bei Landtagswahlen finden, modifizieren Gaines und Crombez diese Befunde. Im Lichte des nun wesentlich längeren Untersuchungszeitraumes (1946 bis 2001) sowie unter Berücksichtigung der Stimmenentwicklungen bei Landtagswahlen nach der deutschen Wiedervereinigung schreiben sie dem ökonomischen Wählen mehr Erklärungskraft zu als zunächst angenommen: „The two large parties appear to gain 1-2 percent of the vote for each 1 percent improvement in GNP under their watch and to lose a comparable amount as their rivals hold national power while the economy grows. "27 Allerdings ziehen Gaines und Crombez aus ihren aggregierten Befunden keine Schlüsse auf das individuelle Wahlverhalten und empfehlen nachdrücklich eine zusätzliche Überprüfung der ermittelten Zusammenhänge anhand von individuellen Umfragedaten. ${ }^{28}$ Ebenfalls anhand von Aggregatdaten untersucht Simone Burkhart ${ }^{29}$ den Einfluss der Bundespolitik auf Landtagswahlentscheidungen von 1976 bis 2002. Sie kommt zu dem Schluss, dass die Verluste der Parteien der Bundesregierung bei Landtagswahlen zunehmen, wenn die Popularität der Bundesregierung seit ihrem Amtsantritt sinkt. Für die zukünftige Forschung fordert sie folglich den Einbezug ökonomischer Variablen, um das Abschneiden der Bundesregierungsparteien bei Landtagswahlen zu erklären.

Zwar gibt es für Deutschland neuere Wahlstudien ${ }^{30}$, welche die Bedeutung der subjektiven Einschätzung der Konjunkturentwicklung auf die Wahlabsicht systematisch und fundiert mit Individualdaten untersucht haben. Allerdings beziehen sich diese ausschließlich auf Wählerpräferenzen im Kontext von Bundestagswahlen. ${ }^{31}$ Eine systematische quantitative Analyse des Zusammenhangs zwischen der subjektiven Wirtschaftseinschätzung und dem Abschneiden der Bundesregierungsparteien bei Landtagswahlen liegt dagegen für individuelles Wahlverhalten im föderalen System Deutschlands bis heute nicht vor.

Um die bisherige Forschungsperspektive zu erweitern, wird in diesem Beitrag die individuelle Landtagswahlabsicht als abhängige Variable modelliert. Es wird angenommen, dass diese Größe davon abhängt, wie die zukünftige Entwicklung der allgemeinen Wirtschafts-

27 Brian J. Gaines / Christophe Crombez, a.a.O., S. 315.

28 Ebenda, S. 316.

29 Simone Burkhart, a.a.O.

30 Jürgen Maier / Hans Rattinger, Economic Conditions and the 1994 and 1998 Federal Elections, in: German Politics, 8. Jg. (1999), S. 33 - 47; Hans Rattinger, Konjunkturentwicklung, Wahrnehmungen der Wirtschaftslage und Parteipräferenzen in Deutschland, 1977 - 1998, in: Markus Klein / Wolfgang Jagodzinski / Ekkehard Mochmann / Dieter Ohr (Hrsg.), 50 Jahre empirische Wahlforschung in Deutschland - Entwicklung, Befunde, Perspektiven, Daten, Wiesbaden 2000, S. 309 - 339; Hans Rattinger / Thorsten Faas, Wahrnehmungen der Wirtschaftslage und Wahlverhalten, 1977 bis 1998, in: Hans-Dieter Klingemann / Max Kaase (Hrsg.), Wahlen und Wähler: Analysen aus Anlass der Bundestagswahl 1998, Wiesbaden 2001, S. 283 - 308; Jürgen Maier I Hans Rattinger, Economic Conditions and Voting Behaviour in German Federal Elections, 1994-2002, in: German Politics, 13. Jg. (2004), S. $201-217$.

31 Vgl. hierzu auch schon die älteren Arbeiten von Hans Rattinger: ders., Allgemeine und persönliche wirtschaftliche Lage als Bestimmungsfaktoren politischen Verhaltens bei der Bundestagswahl 1983, in: Dieter Oberndörfer / Hans Rattinger / Karl Schmitt (Hrsg.), Wirtschaftlicher Wandel, religiöser Wandel, Wertewandel: Folgen für das politische Verhalten in der Bundesrepublik Deutschland, Berlin 1985, S. 183 - 218; ders., Collective and Individual Economic Judgements and Voting in West Germany, 1961 - 1984, in: European Journal of Political Research, 14. Jg. (1986), S. $393-419$. 
lage subjektiv eingeschätzt wird. Damit wird die folgende Leithypothese getestet: Je pessimistischer ein Wähler die zukünftige allgemeine wirtschaftliche Lage in der Bundesrepublik einschätzt, desto eher wird er bei einer Landtagswahl gegen die Parteien der Bundesregierung stimmen.

Um die Robustheit der Befunde sicherzustellen, werden zudem bei jedem Befragten das berichtete Wahlverhalten hinsichtlich der letzen Bundestagswahl und weitere Drittvariablen überprüft. ${ }^{32}$ Damit soll der wohlbekannten Tatsache Rechnung getragen werden, dass aktuelle politische Präferenzen mit vergangenen Wahlentscheidungen zusammenhängen. ${ }^{33}$

\section{Empirischer Test der Economic Voting-Hypothese bei Landtagswahlen}

\subsection{Datenbasis}

Gemäß der im Zentrum stehenden prospektiven Variante des Ansatzes vom ökonomischen Wählen werden Bundesregierungsparteien bei Landtagswahlen überproportional häufig von solchen Wählern nicht wiedergewählt, welche die zukünftige allgemeine Wirtschaftsentwicklung pessimistisch einschätzen. Diese Hypothese soll auf der Basis von Forsa-Bus-Umfragedaten von 1995 bis 2002 empirisch überprüft werden. ${ }^{34}$ Bei den hier analysierten Individualdatensätzen handelt es sich nach unseren Recherchen um die einzigen bundesweiten

32 Der Indikator zur Bundestagswahl-Rückerinnerung ist allerdings nicht unproblematisch. Befragte neigen dazu, vergangenes Wahlverhalten zu vergessen beziehungsweise dieses an das gegenwärtige anzugleichen. Die gleichzeitige Erhebung aktueller und zurückliegender Parteipräferenzen unterschätzt somit systematisch das Ausmaß der Wechselwahl zwischen Bundes- und Landtagswahlen. Vgl. Harald Schoen, Wählerwandel und Wechselwahl - Eine vergleichende Untersuchung, Wiesbaden 2003; ders., Wechselwähler in den USA, Großbritannien und der Bundesrepublik Deutschland: Politisch versiert oder ignorant?, in: ZParl, 35. Jg. (2004), H. 1, S. 99 - 112. Systematische Messfehler auf einer unabhängigen Variablen führen bekanntlich zu einer verzerrten Schätzung des entsprechenden Regressionskoeffizienten. Im vorliegenden Fall wird von einer Verzerrung derart ausgegangen, dass die Modelle den stabilisierenden Effekt der Parteibindungen zu Lasten des ökonomischen Effekts überschätzen.

33 An dieser Stelle wäre auch eine alternative Interpretation denkbar, wonach politische Präferenzen bei zurückliegenden Wahlen die ökonomischen Perzeptionen derart beeinflussen, dass überzeugte Anhänger der Bundesregierungsparteien grundsätzlich optimistischere Wahrnehmungen berichten als Oppositionsanhänger. Träfe dies zu, dann könnte man argumentieren, dass die Wahl der Bundesregierung durch Optimisten weniger Ausdruck ökonomischen Wählens, sondern eher Ausdruck einer starken Parteineigung inklusive Wahrnehmungsbeeinflussung sei. Allerdings postuliert die der Analyse hier zugrunde liegende Theorie des ökonomischen Wählens den Wirkungszusammenhang anders herum. Demnach wird angenommen, dass die subjektiven Wirtschaftswahrnehmungen die individuellen Wahlentscheidungen determinieren (vgl. Anthony Downs, a.a.O.; Michael S. Lewis-Beck / Mary Stegmaier, a.a.O.).

34 Es handelt sich hierbei um werktäglich von der Forsa („Gesellschaft für Sozialforschung und statistische Analyse $\mathrm{mbH}^{\prime \prime}$ ) erhobene Daten, die auf Jahresbasis kumuliert wurden. Die einzelnen Datensätze enthalten Teile aus Mehrthemen-Erhebungen des Forsa-Instituts. Die Interviews wurden jeweils telefonisch mit standardisierten Fragebögen durchgeführt. Dabei wurden die Zielpersonen zufällig ermittelt und befragt. Zu den befragten Individuen liegen in der Regel die folgenden Informationen vor: Rückerinnerung zum Wahlverhalten bei den letzten Bundestags- beziehungsweise Landtagswahlen sowie Wahlabsicht (Sonntagsfrage) bezüglich der kommenden Bundestags- beziehungsweise Landtagswahlen. In diesem Zusammenhang wurden also jeder Person vier verschiedene Fragen gestellt. 
Umfragen, die über einen längeren Zeitraum hinweg individuelles Wahlverhalten auf Bundes- und gleichzeitig auf Landesebene systematisch erfassen. Alle weiterführenden Details zur Datenbasis sind dem Anhang zu entnehmen. Ebenfalls im Anhang befindet sich eine detaillierte Beschreibung der Operationalisierung und Generierung aller hier verwendeten Variablen.

\subsection{Einfacher Zusammenhang zwischen Wirtschaftserwartungen und Landtagswahl- absicht}

Betrachtet man zunächst den einfachen Zusammenhang zwischen der individuellen Landtagswahlabsicht und den subjektiven ökonomischen Erwartungen (vgl. Abbildung 1), so stützen die empirischen Befunde in allen acht Datensätzen die These vom ökonomischen Wählen. Demnach geht mit sinkendem Optimismus gegenüber der zukünftigen allgemeinen Wirtschaftsentwicklung in Deutschland eine klare Abnahme der Abstimmungsbereitschaft zugunsten der Bundesregierungsparteien einher. Als Ablesebeispiel kann der ForsaBus 1996 herangezogen werden (vgl. Abbildung links oben). Hierbei bildet die y-Achse den prozentualen Anteil derjenigen Wähler ab, die für die ausstehende Landtagswahl eine Wahlabsicht zugunsten der amtierenden Bundesregierungsparteien äußerten. Auf der xAchse wurden die Befragten gemäß ihres Pessimismusgrades gegenüber der zukünftigen ökonomischen Entwicklung in drei verschiedene Gruppen eingeteilt. Durch einen Abgleich der jeweiligen Balkenlänge mit der y-Achse zeigen sich für 1996 folgende Befunde: Während 62 Prozent aller optimistischen Wähler in den Interviews angaben, bei der kommenden Landtagswahl für die Bundesregierungsparteien stimmen zu wollen, sank dieser Anteil bei den indifferenten Wählern auf 50 Prozent und bei den pessimistischen Wählern auf nur 35 Prozent. Diese Werte lassen sich direkt von der y-Achse des jeweiligen Schaubildes ablesen. Die übrigen sieben Jahre zeigen dasselbe Muster: Die Balkenlängen, die den Zustimmungsgrad der Wähler zu den Bundesregierungsparteien abbilden, sinken mit zunehmend pessimistischeren ökonomischen Erwartungen der jeweiligen Gruppe.

\subsection{Untersuchungsdesign, Modellentwicklung und statistische Hypothesen}

Mit abnehmendem Optimismus bezüglich der zukünftigen Wirtschaftsentwicklung in der Bundesrepublik sinkt bei den Wählern - unter Kontrolle ihrer vergangenen Bundestagswahlentscheidung sowie weiterer sozialstruktureller Merkmale - die Wahrscheinlichkeit, bei den Landtagswahlen die Parteien der Bundesregierung wiederzuwählen. Die abhängigen Variablen stellen jeweils ein dichotomes Merkmal mit nur zwei möglichen Ausprägungen dar. Für die Analyse von binären Variablen gelten logistische Regressionsmodelle als besonders adäquat. ${ }^{35}$

35 Beim logistischen Regressionsmodell (synonym: „Logit-Modell“) handelt es sich um ein nichtlineares Wahrscheinlichkeitsmodell, anhand dessen die Wahlpräferenzen bei Landtagswahlen in Form von so genannten „Chancenkoeffizienten“ geschätzt wurden. Vereinfacht dargestellt können mit den geschätzten Regressionskoeffizienten für jede beliebige Merkmalskombination der Befragten die Chancen berechnet werden, bei der nächsten Landtagswahl für eine der amtierenden Bundesregierungsparteien zu stimmen. Am einfachsten ist eine Interpretation anhand der Vorzeichen und der relativen Größe der Koeffizienten: Ein negativer Regressionskoeffizient in der 


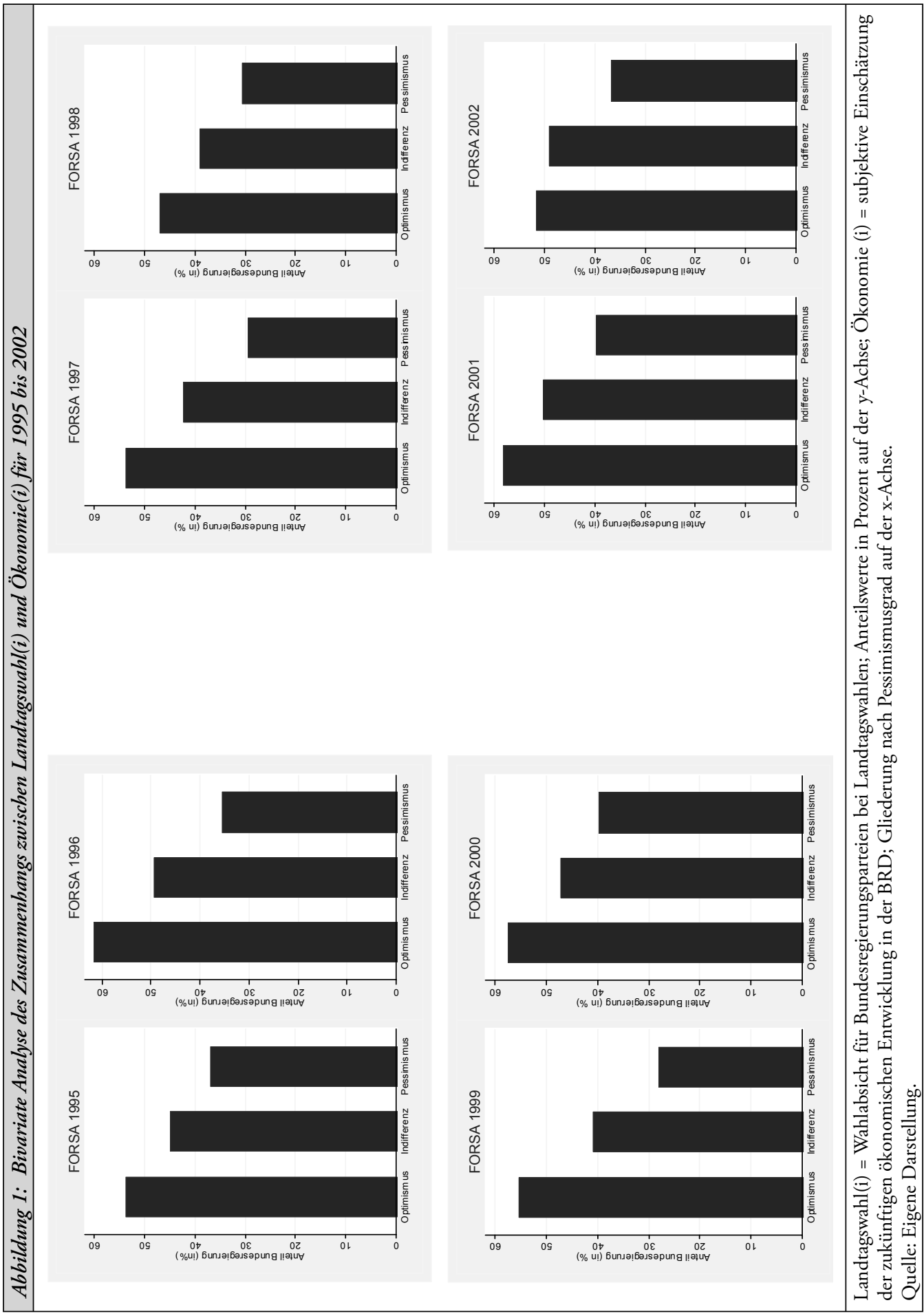


Für die empirische Überprüfung der Hypothese wurde eine Forschungsanordnung gewählt, die häufig auch als „Repeated Survey Design“ beziehungsweise „replikativer Survey” bezeichnet wird. ${ }^{36}$ So stammen die hier analysierten Daten aus Querschnittserhebungen, bei denen wiederholt zu verschiedenen Zeitpunkten anhand weitgehend identischer Fragebögen zufällig ausgewählte Personen aus jeweils unterschiedlichen Stichproben telefonisch befragt wurden. Diese Ausgangssituation ist besonders geeignet, ein und dasselbe statistische Modell anhand von Daten aus mehreren Untersuchungen zu wiederholen und dadurch seine Erklärungskraft zu überprüfen. Das zu schätzende logistische Regressionsmodell lautet wie folgt:

$\ln (\mathrm{W}[$ Landtagswahl $(\mathrm{i})=1]: \mathrm{W}[$ Landtagswahl $(\mathrm{i})=0])=\mathrm{a}+\mathrm{b} 1 \cdot \operatorname{Indifferenz}(\mathrm{i})+\mathrm{b} 2 \cdot$ Pessimismus(i) + b3 . Bundestagswahl(i) + b4 Geschlecht(i) + b5 A Alter(i) + b6 . Bildung(i)

wobei:

Landtagswahl(i) = Sonntagsfrage zur Parteiwahl bei der kommenden Landtagswahl für das i-te Individuum ( 1 = Wahl einer Partei aus den Reihen der amtierenden Bundesregierungskoalition; 0 = Wahl einer Oppositionspartei) als abhängige Variable.

Optimismus(i) = Dummyvariable zur subjektiven Einschätzung der zukünftigen ökonomischen Lage für das i-te Individuum ( 1 = optimistische Einschätzung der allgemeinen wirtschaftlichen Lage; 0 = indifferente beziehungsweise pessimistische Wirtschaftserwartung). Diese Variable stellt die Referenzkategorie dar und wird daher aus den Modellgleichungen ausgeschlossen.

Indifferenz(i) = Dummyvariable zur subjektiven Einschätzung der zukünftigen ökonomischen Lage für das i-te Individuum ( 1 = indifferente Einschätzung der allgemeinen wirtschaftlichen Lage; 0 = optimistische beziehungsweise pessimistische Wirtschaftserwartung). Pessimismus(i) = Dummyvariable zur subjektiven Einschätzung der zukünftigen ökonomischen Lage für das i-te Individuum ( 1 = pessimistische Einschätzung der allgemeinen wirtschaftlichen Lage; 0 = optimistische beziehungsweise indifferente Wirtschaftserwartung).

Bundestagswahl(i) = Rückerinnerung zum Wahlverhalten bei vergangener Bundestagswahl für das i-te Individuum ( 1 = Wahl einer zum Zeitpunkt der Befragung amtierenden Bundesregierungspartei; $0=$ Wahl einer Oppositionspartei).

Geschlecht(i) = Geschlecht des i-ten Individuums ( 1 = männlich; 0 = weiblich $)$.

Alter $(\mathrm{i})=$ Alter (zentriert) des i-ten Individuums.

Bildung(i) = Bildungsniveau des $\mathrm{i}$-ten Individuums ( 1 = hoch; 0 = niedrig).

„W“ bedeutet in diesem Zusammenhang „Wahrscheinlichkeit“; mit „ln“ ist der natürliche Logarithmus des Quotienten W[Landtagswahl(i)=1]: W[Landtagswahl(i)=0] gemeint.

Gruppe der pessimistischen Wähler bedeutet (relativ zu der gewählten Kategorie der optimistischen Wähler) ein Absinken der Abstimmungswahrscheinlichkeit für die amtierenden Bundesregierungsparteien beziehungsweise einen Anstieg der Wechselwahlwahrscheinlichkeit zugunsten der Oppositionsparteien. Das Ausmaß der Veränderung der Wahrscheinlichkeiten ist dabei umso stärker, je höher der Betrag des jeweiligen Regressionskoeffizienten ausfällt. Zum Zwecke einer detaillierten, aber verständlichen Erläuterung der Basiskonzepte des Logit-Modells sei verwiesen auf: Menard Scott, Applied Logistic Regression Analysis, 2. Auflage, Thousand Oaks 2002; Peter Kennedy, A Guide to Econometrics, 5. Auflage, Cambridge 2003.

36 Glenn Firebaugh, Analyzing Repeated Surveys, Thousand Oaks 1997. 
Die Interpretation der geschätzten Koeffizienten erfolgt anhand der Vorzeichen und der relativen Größe zueinander. Dabei können drei Erwartungen formuliert werden:

(1) Der Regressionskoeffizient von „Indifferenz(i)“ hat ein negatives Vorzeichen. In diesem Fall könnte auf eine relativ zur Referenzkategorie: „optimistische Einschätzungen der allgemeinen wirtschaftlichen Lage“ („Optimismus(i)“) niedrigere Wahrscheinlichkeit der Stimmabgabe für die Bundesregierungsparteien derjenigen Personen geschlossen werden, die in die Kategorie „indifferente Wirtschaftseinschätzung“ fallen.

(2) Der Regressionskoeffizient von „Pessimismus(i)“ hat ein negatives Vorzeichen. Träfe dies zu, würden Befragte mit einer pessimistischen ökonomischen Einschätzung eine geringere Wahlwahrscheinlichkeit für die Parteien der Bundesregierung aufweisen als Befragte mit einer optimistischen Wirtschaftseinschätzung (dies wären also alle Personen in der Referenzkategorie „Optimismus(i)“).

(3) Der Regressionskoeffizient der unabhängigen Variable „Indifferenz(i)“ ist im Vergleich zum Koeffizienten der unabhängigen Variable „Pessimismus(i)“ signifikant größer (formaler: „Indifferenz(i)“ > „Pessimismus(i)“). Zeigte sich diese Erwartung in den Daten, könnte dies als ein Hinweis darauf gedeutet werden, dass Individuen mit einer pessimistischen subjektiven Wirtschaftsprognose eine niedrigere Abstimmungswahrscheinlichkeit zugunsten der Parteien der Bundesregierung bei den Landtagswahlen erkennen lassen als Befragte, die für die Zukunft keine wesentliche Veränderung der allgemeinen Wirtschaftslage vermuten. Zum Zwecke der Überprüfung dieser Annahme wurde jeweils in allen acht Datensätzen ein Signifikanztest („Wald-Test“) auf Gleichheit der beiden Regressionskoeffizienten durchgeführt (Nullhypothese: „Indifferenz(i)“ = „Pessimismus(i)“).

\section{Empirische Befunde}

Das hier beschriebene Modell wurde auf identische Art und Weise in allen acht Datensätzen geschätzt. Dabei erfolgten die Berechnungen jeweils getrennt nach West- und Ostdeutschland. Die folgende Tabelle bildet die empirischen Befunde hierzu ab $b^{37}$ :

37 Die Modelle wurden zusätzlich mit einer alternativen Spezifikation repliziert. Hierbei wurde anstatt der Bundestagswahl-Rückerinnerung eine identisch konstruierte Variable zur LandtagswahlRückerinnerung als verzögerte endogene Variable verwendet. Es ergeben sich identische Schlussfolgerungen. Die Economic Voting-Effekte sind ausnahmslos signifikant und zeigen gemäß der statistischen Hypothesen in die erwartete Richtung. Es wird an dieser Stelle aus inhaltlichen Erwägungen auf die explizite Darstellung dieser Befunde verzichtet, da das Hauptinteresse der Interdependenz zwischen Bundes- und Landesebene und nicht etwa den Zusammenhängen zwischen Landtagswahlen gilt. Diese zusätzlichen Resultate können bei den Autoren angefordert werden. 


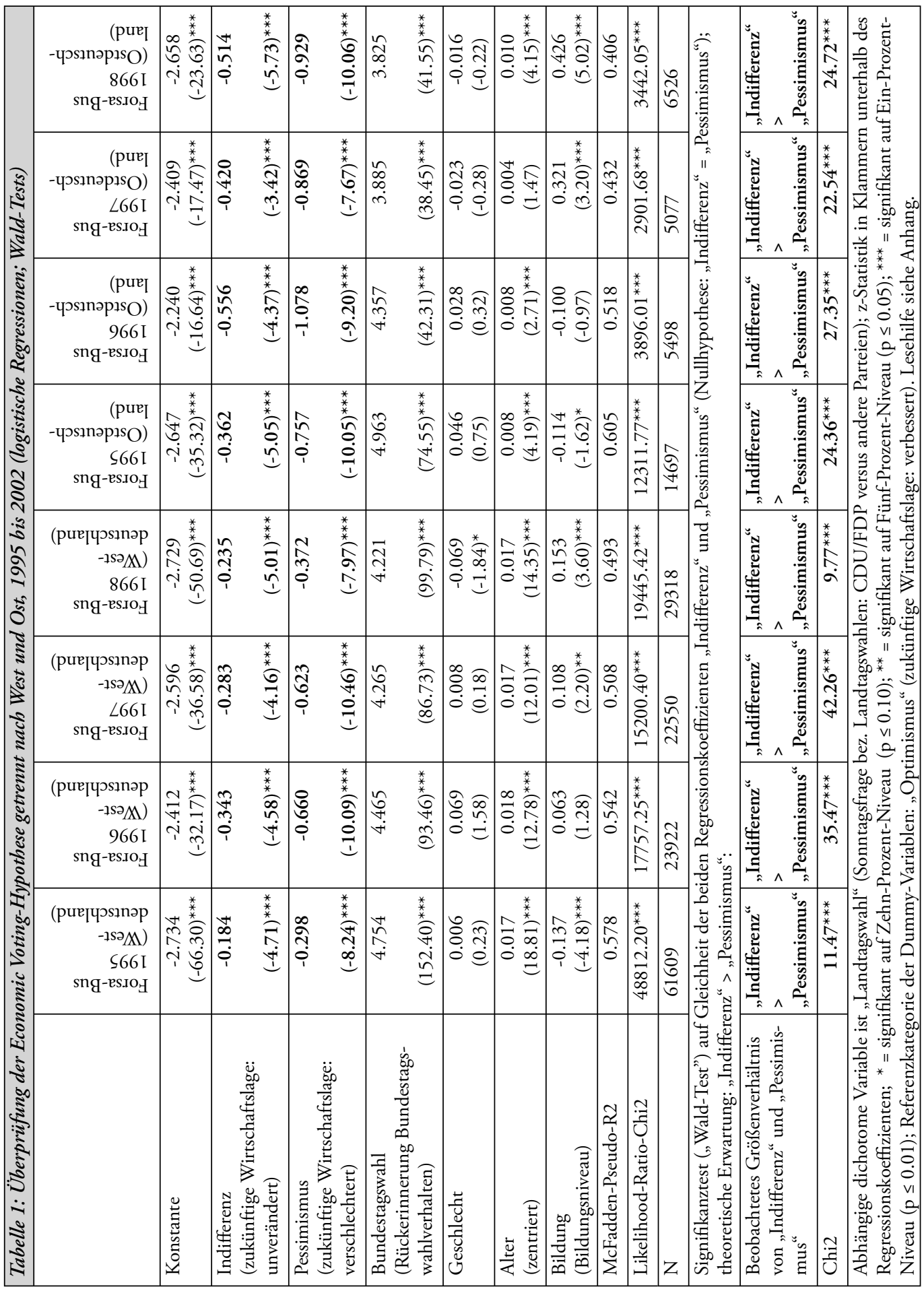




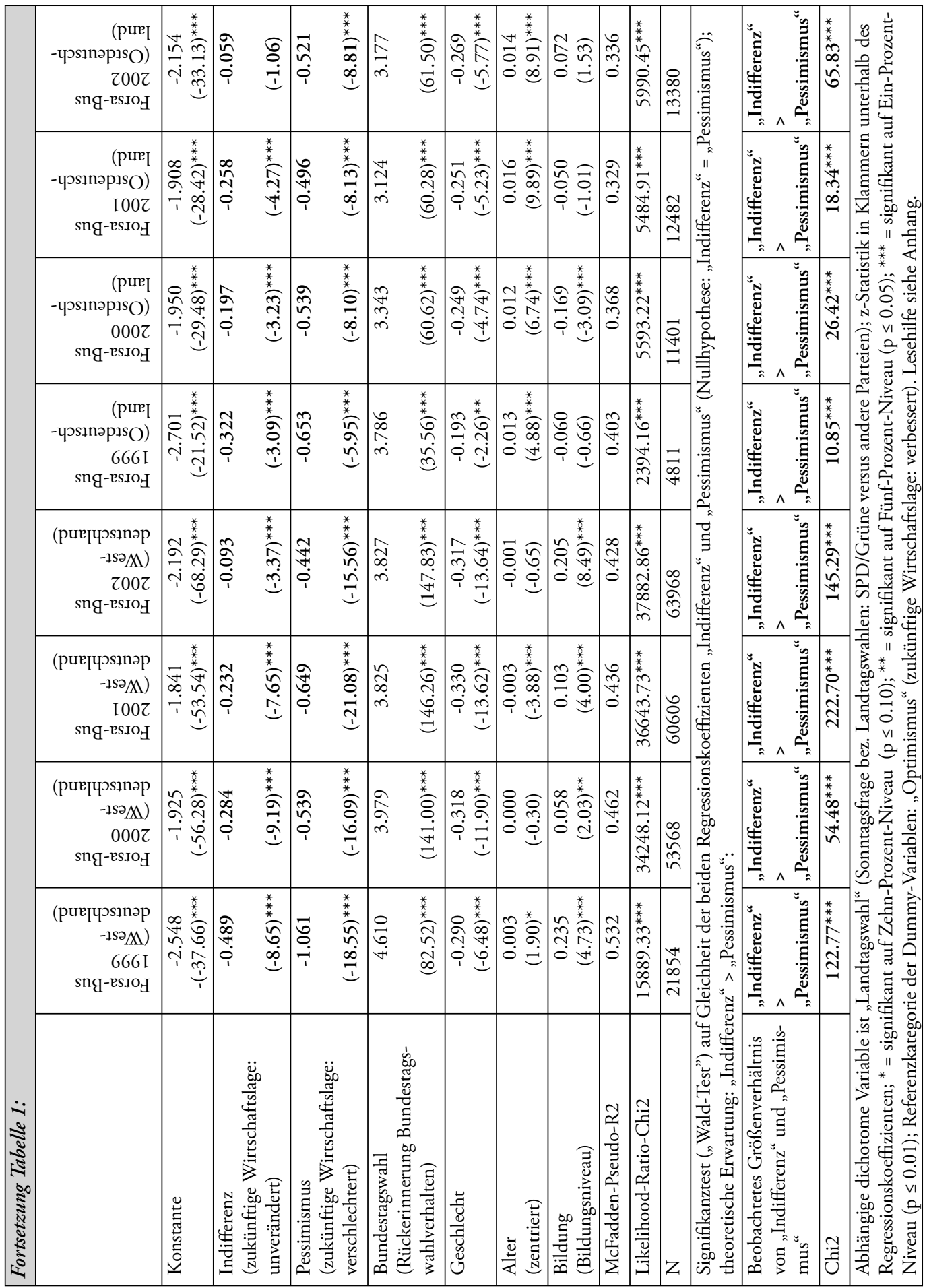


Bei allen 16 Replikationen des logistischen Regressionsmodells finden sich jeweils - bis auf eine einzige Ausnahme - die drei theoretischen Erwartungen empirisch bestätigt. Hierbei ist es am einfachsten, die Vorzeichen und die relative Größe der Regressionskoeffizienten zu interpretieren: Die Koeffizienten von „Indifferenz(i)“ und „Pessimismus(i)“ weisen durchgehend das erwartete negative Vorzeichen auf und sind in der Regel auf dem Ein-ProzentNiveau signifikant $(\mathrm{p} \leq 0.01) .{ }^{38}$ Demnach sinken im Vergleich zu optimistischen Befragten bei indifferenten und pessimistischen Wählern die Wahrscheinlichkeiten, bei den Landtagswahlen die Bundesregierungsparteien zu wählen, ab. Ebenso lässt sich im Rahmen der Wald-Tests in allen Fällen eine signifikante Differenz zwischen den beiden Regressionskoeffizienten „Indifferenz(i)“ und „Pessimismus(i)“ in die erwartete Richtung feststellen ( $\mathrm{p} \leq$ 0.01). Demzufolge liegt bei pessimistischen Wählern eine noch geringere Wahrscheinlichkeit vor, bei Landtagswahlen zugunsten der Parteien der Bundesregierung zu votieren, als bei Befragten, die gegenüber der zukünftigen ökonomischen Entwicklung indifferent sind. In Bezug auf die Referenzkategorie der Optimisten ist somit das Ausmaß des Absinkens der Wahrscheinlichkeiten bei den Pessimisten stärker als bei indifferenten Wählern. ${ }^{39}$ Dieser Befund erweist sich sowohl regional (alte und neue Bundesländer) als auch über die unterschiedlichen Regierungskoalitionen hinweg (CDU/CSU/FDP-Bundesregierung und SPD/ Grüne-Bundesregierung) als stabil.

Alle 16 Modelle haben zudem einen akzeptablen bis guten Gesamt-Fit. ${ }^{40}$ Auch deuten in jedem einzelnen der 16 Modelle die ermittelten LR-Chi ${ }^{2}$-Werte auf einen signifikanten Modellfit hin $(\mathrm{p} \leq 0.01)$. Zusammenfassend kann somit die Hypothese des prospektiven ökonomischen Wählens auf der Individualebene statistisch bestätigt werden.

Als inhaltliches Hauptergebnis ist die Erkenntnis festzuhalten, dass die wahrgenommene Entwicklung der allgemeinen wirtschaftlichen Lage im individuellen Wahlkalkül eine wich-

38 Einzige Ausnahme: Im Forsa-Bus 2002 (vgl. Tabelle 1 Fortsetzung) weist der Regressionskoeffizient der unabhängigen Variable „Indifferenz(i)“ beim Modell für Ostdeutschland zwar das richtige negative Vorzeichen auf, ist jedoch auf keinem der drei konventionellen Niveaus signifikant von Null verschieden.

39 Um eine präzise quantitative Aussage hinsichtlich der mittleren Effektstärke formulieren zu können, wurden zunächst für die jeweiligen Subgruppen die durchschnittlichen partiellen Regressionskoeffizienten (durch einfache Mittelwertbildung) berechnet: -0.268 für indifferente Wähler im Westen, -0.336 für Indifferente im Osten, -0.581 für pessimistische Wähler im Westen sowie -0.730 für Pessimisten im Osten. In einem zweiten Schritt wurden die so ermittelten Werte durch einfaches Exponieren in durchschnittliche Chancenverhältnisse (sog. „Odds-Ratios“) umgerechnet; Folgendes kann festgehalten werden: Bei einem indifferenten Wähler ist die Chance der Wahl von Bundesregierungsparteien im Mittel nur 0.769-mal (im Westen) beziehungsweise 0.723-mal (im Osten) so groß wie die entsprechende Chance eines Optimisten. Dagegen ist bei einem Pessimisten die Chance bei Landtagswahlen für die Bundesregierungsparteien zu stimmen im Durchschnitt lediglich 0.572-mal (im Westen) beziehungsweise 0.491-mal (im Osten) so groß wie die eines optimistischen Wählers. Dies bedeutet: Mit zunehmendem Pessimismus gegenüber der allgemeinen ökonomischen Situation in der Bundesrepublik steigt bei Landtagswahlen die Wahrscheinlichkeit der Wechselwahl zuungunsten der Bundesregierungsparteien.

40 Als zentrale Maßzahl für die Beurteilung der Güte der Gesamtmodelle wurde jeweils das Pseudo$\mathrm{R}^{2}$ nach McFadden herangezogen. Hierbei wurden Werte zwischen 0.329 (vgl. Tabelle 1: ForsaBus 2001 für Ostdeutschland) und 0.605 (vgl. Tabelle 1: Forsa-Bus 1995 für Ostdeutschland) erreicht. Bei der Berechnung von McFadden-Pseudo-R ${ }^{2}$ wird jeweils der Quotient der Wahrscheinlichkeit des gegebenen Modells zur Wahrscheinlichkeit eines Modells gebildet, bei dem alle Parameter außer der Konstante auf null gesetzt sind: Pseudo- $\mathrm{R}^{2}=1-\ln \left(\mathrm{L}_{1}\right): \ln \left(\mathrm{L}_{0}\right)$. 
tige Rolle spielt. Geht es der Wirtschaft schlecht und nehmen die Wähler dies auch entsprechend wahr, so sinkt unter den Pessimisten die Unterstützung für die amtierende Bundesregierung bei anstehenden Landtagswahlen signifikant ab, und eine Wahlentscheidung zugunsten der Oppositionsparteien wird wahrscheinlicher.

Die hohe Relevanz der subjektiven Einschätzung der zukünftigen ökonomischen Entwicklung für die Wahlabsicht wird dabei schon anhand einfacher bivariater Betrachtungen deutlich (vgl. Abbildung 1). So wollte beispielsweise 1999 eine klare Mehrheit von 55 Prozent aller optimistischen Wähler bei den kommenden Landtagswahlen für die Bundesregierungsparteien stimmen. Dieser Anteil betrug in der Gruppe der indifferenten Wähler lediglich 41 Prozent und sank bei pessimistischen Wählern auf einen Wert von nur noch 28 Prozent Zustimmung für die Bundesregierungsparteien ab. Die anderen Jahre zeigen dasselbe Muster. Die durchgeführten logistischen Regressionsmodelle unter Einbezug einer Reihe von Kontrollvariablen untermauern die Bedeutung der Einschätzung der zukünftigen Wirtschaftslage für die Landtagswahlabsicht. So ist bei einem Pessimisten die Chance, bei Landtagswahlen für die Bundesregierungsparteien zu stimmen, im Durchschnitt nur noch etwa halb so groß wie die eines optimistischen Wählers: das 0.57-fache in Westdeutschland beziehungsweise das 0.49-fache in Ostdeutschland. ${ }^{41}$

Insgesamt ist mit sinkendem Optimismus gegenüber der zukünftigen allgemeinen Wirtschaftslage in Deutschland eine klare Abnahme der Wahlbereitschaft zugunsten der Bundesregierungsparteien erkennbar, wobei die vorliegenden Befunde zusätzlich darauf hinweisen, dass in der Mehrheit der betrachteten Jahre der Effekt des ökonomischen Wählens im Osten stärker ist als in Westen (vgl. Tabelle 1). ${ }^{42}$ Als mögliche Erklärungen kommen die höhere Wechselwahlbereitschaft und generell instabilere Parteibindungen in den neuen Bundesländern in Frage. ${ }^{43}$ Auch die Regressionsbefunde deuten in diese Richtung: In sieben der acht untersuchten Jahre fallen die Effektstärken zur Bundestagswahl-Rückerinnerung im Osten geringer aus als im Westen. ${ }^{44}$ Somit scheint die stabilisierende Wirkung des vergangenen Wahlverhaltens in den alten Bundesländern stärker ausgeprägt zu sein als in den neuen. Zukünftige Forschungsarbeiten könnten die Ursachen dieser Unterschiede genauer untersuchen.

\section{5. Ökonomisches Wählen: mit Mikrodaten belegt}

Die vorliegenden Ergebnisse stehen im Einklang mit den bisherigen Individualdatenanalysen zum ökonomischen Wählen in Deutschland, insbesondere mit den fundierten Untersuchungen von Hans Rattinger und seinen Mitarbeitern ${ }^{45}$, die ebenfalls die Bedeutung der

41 Vgl. hierzu die Ausführungen in Fußnote 39.

42 Die Effektstärke lässt sich an den Beträgen der Regressionskoeffizienten ablesen. So sind beispielsweise bei pessimistischen Wählern in Tabelle 1 die jeweiligen Beträge in Ostdeutschland in der Mehrheit der betrachteten Jahre höher als in Westdeutschland (Ausnahme: Jahr 1999 mit 1.061 im Westen versus $0.653 \mathrm{im}$ Osten und Jahr $2001 \mathrm{mit} 0.649 \mathrm{im}$ Westen versus $0.496 \mathrm{im}$ Osten).

43 Cornelia Weins, The East German Vote in the 1998 General Election, in: German Politics, 8. Jg. (1999), S. $66-68$.

44 Nur im Jahr 1995 ist der Betrag des Regressionskoeffizienten zur Bundestagswahl-Rückerinnerung in Ostdeutschland höher: $4.754 \mathrm{im}$ Westen versus $4.963 \mathrm{im}$ Osten (vgl. Tabelle 1).

45 Jürgen Maier / Hans Rattinger, a.a.O. (2004); Hans Rattinger, a.a.O.; Hans Rattinger / Thorsten Faas, a.a.O. 
Perzeption der allgemeinen wirtschaftlichen Lage für das Wahlverhalten bei den zurückliegenden Bundestagswahlen herausstreichen. Hinzu kommt, dass nun erstmalig anhand von Mikrodaten gezeigt werden konnte, dass die Einschätzung der ökonomischen Situation nicht nur bei Wahlen auf der Bundesebene relevant, sondern auch für das Abschneiden der Bundesregierungsparteien bei Landtagswahlen verantwortlich ist. Es kann nunmehr davon ausgegangen werden ${ }^{46}$, dass ökonomisches Wählen nach der deutschen Wiedervereinigung eine hohe Erklärungskraft besitzt. ${ }^{47}$ In der laufenden Kontroverse, ob Landtagswahlen eher eine Art Referendum für die Regierungskoalition auf Bundesebene oder Wahlen nach rein regionalen Gesichtspunkten darstellen, stützen unsere Ergebnisse die These von Frank Dekker und Julia von Blumenthal. ${ }^{48}$ Wahlen in den Bundesländern sind nach wie vor zu einem beträchtlichen Grad Stimmungsentscheidungen für oder gegen die Bundesregierung und können - im Gegensatz zu der These von Daniel Hough und Charlie Jeffery ${ }^{49}$ - nicht als reine Regionalwahlen betrachtet werden, die einer typisch landeseigenen Logik folgen.

Die Resultate dürften nicht nur für die quantitative Wahlforschung, sondern auch für eine breitere Öffentlichkeit von Interesse sein. Ökonomie und politische Wahlen sind eng miteinander verbunden. Dazwischen geschaltet liegen die sensiblen, durch die politischen Akteure beziehungsweise die Medien prinzipiell beeinflussbaren, individuellen Wahrnehmungen dieser Entwicklungen. Auf der einen Seite versuchen Parteien durch geschickte Informationsauslegungen, günstige Szenarien im Hinblick auf ihre zukünftigen Wahlergebnisse zu entwerfen. Auf der anderen Seite stehen Wähler, die sich über verschiedene unabhängige Quellen informieren und sich ein Urteil über die wirtschaftliche Situation verschaffen. Kommen sie zu einer pessimistischen Einschätzung, assoziieren sie dies häufig mit einer fehlgeschlagenen Wirtschaftspolitik der amtierenden Bundesregierungsparteien, was sodann in den individuellen Landtagswahlpräferenzen seinen entsprechenden Ausdruck findet.

46 In Übereinstimmung mit Brian J. Gaines / Christophe Crombez, a.a.O., S. 315.

47 An dieser Stelle soll kurz auf mögliche Probleme hinsichtlich des von uns untersuchten Zeitraums zwischen 1995 und 2002 eingegangen werden: Ein denkbarer Einwand wäre, dass während des Beobachtungszeitraums besondere Umstände herrschten (so war beispielsweise die deutsche Wirtschaft von 1995 bis 2002 von einer relativ schlechten Konjunkturlage geprägt) und es daher nur schwer möglich ist, die empirischen Befunde auf andere Zeiträume, so etwa die Zeit vor der Wende, zu generalisieren. Dagegen kann man einwenden, dass es sich beim ökonomischen Wählen um eine allgemeine Hypothese über individuelles Wählerverhalten handelt, die unabhängig von einem spezifischen Kontext ihre Wirkung beansprucht. So ist es auch angesichts eines wirtschaftlichen Aufschwungs nicht unplausibel, dass es weiterhin viele pessimistische Wähler gibt, die bei Landtagswahlen gegen die Bundesregierungsparteien stimmen. Die individuell wahrgenommene Wirtschaftsentwicklung auf der Mikroebene einerseits und die Verläufe von makroökonomischen Indikatoren andererseits können beträchtlich divergieren. Zudem hindert jedoch neben diesem theoretischen Argument die Datenlage daran, Analysen für größere Zeiträume durchzuführen. Die in den Modellen hier verwendeten Indikatoren im Forsa-Bus wurden erst seit 1995 erhoben. Somit können keine identischen Berechnungen für den Zeitraum vor der deutschen Wiedervereinigung durchgeführt werden. Dementsprechend beschränken sich die Schlussfolgerungen in erster Linie auf den Zeitraum nach der Wende.

48 Frank Decker / Julia von Blumenthal, Die bundespolitische Durchdringung der Landtagswahlen. Eine empirische Analyse von 1970 bis 2001, in: ZParl, 33. Jg. (2002), H. 1, S. 144 - 164.

49 Daniel Hough / Charlie Jeffery, Landtagswahlen: Bundestestwahlen oder Regionalwahlen?, in: ZParl, 34. Jg. (2003), H. 1, S. $79-94$. 
Für zukünftige Forschungsarbeiten ergibt sich daraus der Anknüpfungspunkt, den Zusammenhang von subjektiven Perzeptionen der wirtschaftlichen Lage und den tatsächlichen Verläufen von objektiven makroökonomischen Indikatoren systematisch zu untersuchen. Des Weiteren bietet sich an, den Einfluss der subjektiven Wirtschaftswahrnehmung auf Wahlen in Abhängigkeit der zyklischen Verläufe von der Regierungspopularität zu analysieren. ${ }^{50}$ So wäre in diesem Zusammenhang die Hypothese zu testen, wonach die zeitliche Position im Bundestagswahlzyklus die Effektstärke des ökonomischen Indikators beeinflusst. Außerdem wären differenziertere Analysen einzelner Bundesländer interessant, die kurz vor einer Landtagswahl stehen. Dabei wäre zu prüfen, ob aufgrund des präsenteren Landtagswahlkampfes mehr regionale Faktoren in die individuelle Wahlabsicht einfließen und gleichzeitig der Effekt der wahrgenommenen Wirtschaftsentwicklung schwächer wird.

\section{Anhang}

\section{Datenquellen}

Als Datenbasis dient der Forsa-Bus des datenerhebenden Instituts „Forsa Gesellschaft für Sozialforschung und statistische Analyse mbH" (Primärforscher). Die entsprechenden Datensätze wurden vom Zentralarchiv (ZA) für empirische Sozialforschung an der Universität zu Köln zwecks Analysezwecken zur Verfügung gestellt. Hinsichtlich einer Replikation können die Daten jederzeit beim ZA Köln beantragt werden. Konkret handelt es sich dabei um die folgenden Studien: ZA2983 (Forsa-Bus 1995), ZA2984 (Forsa-Bus 1996), ZA2985 (Forsa-Bus 1997), ZA3162 (Forsa-Bus 1998), ZA3289 (Forsa-Bus 1999), ZA3486 (Forsa-Bus 2000), ZA3675 (Forsa-Bus 2001), ZA3909 (Forsa-Bus 2002). Eine vollständige Dokumentation der hier durchgeführten Datenanalyse kann jederzeit bei den Autoren angefordert werden.

\section{Details zur Operationalisierung und Variablenbildung}

Ausgehend von den Forsa-Bus-Originalitems wurden die folgenden Variablen konstruiert:

- „Landtagswahl(i)“ (Sonntagsfrage bezüglich der kommenden Landtagswahlen für das i-te Individuum) als dichotome abhängige Variable wurde auf der Basis des entsprechenden Items zur Wahlabsicht generiert und im Lichte unserer Fragestellung codiert als:

$0=$ Befragter würde, wenn am nächsten Sonntag Landtagswahlen wären, für eine Partei aus den Reihen der Opposition stimmen (Bei Befragungen, die zeitlich vor der Bundestagswahl am 27. September 1998 durchgeführt wurden: SPD; Grüne; PDS; sonstige Parteien. Nach dem 27. September 1998: CDU/CSU; FDP; PDS; sonstige Parteien).

1 = Befragter würde, wenn am nächsten Sonntag Landtagswahlen wären, eine der beiden Parteien aus der amtierenden Bundesregierungskoalition wählen (vor dem Bundestagswahltermin am 27. September 1998 handelte es sich hierbei um die Parteien CDU/CSU und FDP. Nach dem 27. September 1998: SPD und Grüne). Folgende Angaben wurden aus der weiteren Betrachtung ausgeschlossen: „,bin nicht wahlberechtigt“", „gehe nicht wählen“, „weiß nicht“ oder „keine Angabe“.

- Die zentrale unabhängige Variable „Ökonomie(i)“ (subjektive Einschätzung der zukünftigen Wirtschaftslage in Deutschland für das i-te Individuum) bildet den individuellen Grad an Pessimismus hinsichtlich der zukünftigen allgemeinen Wirtschaftsverhältnisse ab. Personen mit den Antwortkategorien „weiß nicht“ beziehungsweise „keine Angabe“ wurden aus der Analyse ausgeschlossen. Um logistische Regressionsmodelle rechnen zu können, wurde auf Basis dieser kategorialen Wirkgröße in jedem der acht Datensätze ein Set von Dummy-Variablen gebildet:

- „Optimismus(i)“": 1 = Befragter gibt an, die wirtschaftlichen Verhältnisse in Deutschland würden sich in den kommenden Jahren verbessern; 0 = indifferente beziehungsweise pessimistische Erwartungen.

50 William L. Miller / Myles Mackie, a.a.O. 
- „Indifferenz(i)“: 1 = Befragter gibt an, die wirtschaftlichen Verhältnisse würden in den kommenden Jahren unverändert bleiben; 0 = optimistische beziehungsweise pessimistische Erwartungen.

- „Pessimismus(i)“: 1 = Befragter gibt an, die wirtschaftlichen Verhältnisse würden sich in den kommenden Jahren verschlechtern; 0 = optimistische beziehungsweise indifferente Erwartungen.

Die anderen Kontrollvariablen wurden wie folgt gebildet:

- Die binär codierte Variable „Bundestagswahl(i)“ (Rückerinnerungsfrage zum Abstimmungsverhalten bei der letzten Bundestagswahl für das i-te Individuum):

$0=$ Befragter gibt an, bei der letzten Bundestagswahl für eine Partei aus den Reihen der Opposition gestimmt zu haben (Wurden die Befragten vor dem 27. September 1998 interviewt: SPD; Grüne; PDS; sonstige Parteien. Danach: CDU/CSU; FDP; PDS; sonstige Parteien).

1 = Befragter gibt im Interview an, bei der letzten Bundestagswahl eine zum Zeitpunkt der Befragung amtierende Bundesregierungspartei gewählt zu haben (Wenn die Befragung vor dem 27. September 1998 stattfand: CDU/CSU beziehungsweise FDP. War dagegen der Befragungstermin nach der Bundestagswahl am 27. September 1998: SPD beziehungsweise Grüne). Personen mit den Angaben „habe nicht gewählt“, „war nicht wahlberechtigt“, „weiß nicht mehr“ oder „keine Angabe“ wurden aus der Analyse ausgeschlossen.

- Die Variable „Geschlecht(i)“ (Geschlecht des i-ten Individuums) mit den Ausprägungen: 1 = männlich; 0 = weiblich.

- Die Altersvariable „Alter(i)“ (zentriertes Alter des i-ten Individuums).

- Die Variable „Bildung(i)“ (Bildungsniveau des i-ten Individuums) mit der Codierung: 1 = hohes Bildungsniveau (Fachhochschulreife; allgemeine oder fachgebundene Hochschulreife; Fachhochschul- oder Hochschulabschluss); $0=$ niedriges Bildungsniveau (ohne Hauptschulabschluss; Hauptschulabschluss; Realschulabschluss; Abschluss der Polytechnischen Oberschule). Befragte mit der Codierung ,anderer Schulabschluss“ wurden in der Untersuchung nicht berücksichtigt.

\section{Lesehilfe für Tabelle 1}

Die Werte in Tabelle 1 wurden auf Grundlage der präsentierten Gleichung geschätzt. Lesebeispiel, Spalte „Forsa-Bus 1995 (Westdeutschland)“: Der Koeffizient b1 (indifferente Wähler) wurde mit -0.184 , der Koeffizient b2 (pessimistische Wähler) mit -0.298 geschätzt. Dies bedeutet, dass die „logarithmierten Chancen" bei den Landtagswahlen für die Bundesregierung zu stimmen (der Ausdruck auf der linken Seite der Gleichung) bei den indifferenten beziehungsweise pessimistischen Wählern, gegenüber der Referenzkategorie der optimistischen Wähler, jeweils um diese Beträge sinken. „Logarithmierte Chancen" lassen sich mathematisch in Wahrscheinlichkeiten transformieren. Aus der Mathematik ist bekannt, dass „logarithmierte Chancen“ und Wahrscheinlichkeiten in einem positiven Zusammenhang stehen. Sinken „logarithmierte Chancen“, verringern sich auch die entsprechenden Wahrscheinlichkeiten.

Die Koeffizienten der indifferenten und der pessimistischen Wähler haben jeweils ein negatives Vorzeichen und sind signifikant. Dies bedeutet, dass bei beiden Wählergruppen, gegenüber der Referenzkategorie der optimistischen Wähler, die Wahrscheinlichkeiten, die Bundesregierungsparteien zu wählen, sinken. Damit sind zwei notwendige Bedingungen für die Gültigkeit der Hypothese des prospektiven ökonomischen Wählens erfüllt. Entsprechend wurden diese Befunde schwarz eingefärbt (Bestätigung der Hypothese).

Die dritte Bedingung postuliert, dass die Wahrscheinlichkeit bei pessimistischen Wählern, im Vergleich mit indifferenten Wählern, stärker sinkt. Dieser Befund ist in der Zeile „Beobachtetes Größenverhältnis von ,Indifferenz' und ,Pessimismus'“ dokumentiert. Es zeigt sich im gewählten Beispiel, dass der Koeffizient der indifferenten Wähler mit -0.184 größer ist als der Koeffizient -0.298 der pessimistischen Wähler. Folglich kann auch die dritte Bedingung, Indifferenz > Pessimismus, empirisch bestätigt werden. Die Wahrscheinlichkeit, die Bundesregierungsparteien zu wählen, sinkt, bezogen auf die Referenzkategorie der optimistischen Wähler, bei pessimistischen Wählern stärker als bei indifferenten Wählern. Auch dieser Befund wurde schwarz eingefärbt als Hinweis darauf, dass sich die geforderte Bedingung empirisch bestätigen lässt. 